\title{
Prolong Lifespan of Wireless Sensor Network with Optimized Information Compression Algorithm and Magnetic Resonant Concept
}

\author{
Manish Bhardwaj $^{1 *}$ and Anil Ahlawat ${ }^{2}$ \\ ${ }^{1}$ SRM IST, Computer Science and Engineering, Modinagar, Ghaziabad, India \\ ${ }^{2}$ KIET, Computer Science and Engineering, Muradnagar, Ghaziabad, India \\ ${ }^{*}$ Corresponding author
}

\begin{abstract}
Remote sensor systems maintain numerous applications in various fields. Sparing vitality in such systems is continuously a basic issue that should be considered to delay the network lifespan. Bunching in the systems is additionally considered as a vitality sparing directing strategy. This manuscript, propose the two technique by which the vitality problem of a remote sensor can be resolved. In first Strategy, with the help of wireless power transfer recharge all the node of the network and in the second strategy a new information gathering strategy, called firmness technique, keeping in mind the end goal to altogether diminish information transmitting among grouped systems for vitality sparing reason. In the strategy, Wavelet essential is utilized in each group to pack information to be sent to the base-station (BS). At the BS, all the detecting information can be remade in light of the compacted tests gathered from groups. We additionally investigate what's more, plan the aggregate vitality utilization for information transmitting between sensors in the systems and from the systems to the BS. Reproduction comes about are given to illuminate our examination and to propose ideal cases for the system to expend the slightest control. In this manuscript, new proposed technique shows the results with various performance parameters like, power consumption, network lifespan in Inter and Intra transmissions.
\end{abstract}

Keywords—power consumption; LEACH; base Station; vitality

\section{INTRODUCTION}

Late years, much research contemplates in remote sensor systems take a great deal of consideration since the systems have numerous applications. Following articles or identifying occasions are exceptionally normal applications in various fields. Sensors are sent haphazardly in a backwoods to recognize fire. They can be sent in a detecting field to save individuals or on the other hand to distinguish oil spill. In different cases, sensors are additionally sent to assemble scalar maps for information examination purposes. The maps are manufactured in light of crude information gathered from all sensors in the detecting fields. WSNs are inserted with distinctive topologies. They could be dynamic or settled structures contingent upon the reasons for such systems. Group based, tree-based, babble based, arbitrary walk, and so forth are extremely regular information accumulation techniques applying in the systems. Every technique has unmistakable focuses to be used for particular applications. Remote associations have a few alternatives including Bluetooth, Wi-Fi, $\mathrm{RF}$ in which they are appropriate in applications.

Grouping calculations are considered as a vitality sparing information accumulation strategies in the systems. K-implies, Drain is regular a technique to separate the system into bunches. $\mathrm{K}$-implies enhances the transmitting separations between bunch heads (CHs) and non- $\mathrm{CH}$ sensors to diminish vitality utilization for sensors. Filter picks CHs based on stochastic hypothesis to adjust vitality in the system since the greatest work stack dependably falls on CHs. Numerous other grouping calculations have demonstrated their vitality effective focuses for WSNs. Groups could be orchestrated unequally with a specific end goal to adjust the devoured vitality between bunches since the ones near the BS dependably spend more vitality than the one more remote.

In this paper, we apply Wavelet change hypothesis for bunching in WSNs to pack detecting information in each group In a bunched arrange, each group has one bunch head $(\mathrm{CH})$ also, the rest are non- $\mathrm{CH}$ sensors. All non- $\mathrm{CH}$ sensors send their own particular information to the $\mathrm{CH}$ they have a place with. This $\mathrm{CH}$ increases the gathered information including its own particular information to Wavelet coefficients also, just sends the huge changed coefficients to the base station (BS) or the information handling focus. The BS gathers the extensive coefficients from all groups following that way and recreates all information from the groups to have the capacity to assemble a scalar guide. We additionally propose distinctive approaches to forward the changed coefficients from $\mathrm{CHs}$ to the BS; the coefficients are sent straightforwardly to the BS or transferred between other $\mathrm{CHs}$ to at long last achieve the BS. Vitality utilization for information transmission in the systems is detailed.

The rest of this paper is sorted out as takes after. Issue Formulation and Energy Consumption Analysis are tended to in Section II and III, separately. Reproduction comes about are given in Section IV. At last, Conclusions and future work are in Section V.

\section{RELATED WORK}

Xiaoqin Chen et al [1] Add knowledge transfer of data, which was hired to use the data rate and delay gloves hobby wait queue with a given weak low electrical current to make 
better use of its channels are also offered awareness of traffic protocol for a particular mobile network (CARM). CARM implemented method for data transmission lines, propagation speed in order to prevent data transfer speed road links that do not match. CARM It was discussed and developed a network of only IEEE $802.11 \mathrm{~b}$. However, it is applied to the rates of multinetwork Ad Hoc.

J. M. Kim et al. [2] this paper improves the LEACH protocol with the help of cluster head election method. In this paper fuzzy logic technique is used and with the help of this technique, network chooses the cluster head so that lifetime of the network gets increased.

Manish et al. [3] describe the concept of reduction of power consumption in communication process. This manuscript describes the RECAODV algorithm which minimizes the energy consumption in the network and increase the lifetime of network. It also explain the concept of wireless power transfer between the nodes of the network.

Manish et al. [4] this paper apply a wireless power transfer and Backpressure Technique to reduce the power problem of the network nodes. This paper shows the simulation results by using the energy conservation algorithm. This paper increased the significant lifetime of the network. Link utilization, route maintenance and reduce overhead is the main objective of this paper.

Manish et al. [5] this paper brief the cooperative communication in different nodes which communicate with same data at a single instant of time. This paper uses the optimum relay nodes selection technique in $\mathrm{CC}$ network and with the help of this technique, it reduces the power consumption of the network.

Sotiris Nikoletseas et al. [6] this paper focus on wireless charging of the portable de-vices for this it is work on two different types of protocols one perform wireless charging, evaluate the charging efficiency, and second maintain the balances of the chargers. This paper evaluates the results in real time environment.

Benard Mumo Makaa et al. [7] this paper simply demonstrates the application of solid state tesla coils. According to the results, this paper shows that tesla coils are highly capable of generating high voltage, high-frequency waveforms. It also shows that tesla coils are designed for wireless power transfer.

Xiao Lu et al. [8] this paper discussed the issues related to wireless power charging and development of a commercial product that using this concept of wireless charging. It shows the wireless charging standards and latest network applications which are used in the market. It also discusses the challenges to implementing the wireless charging concept.

\section{WIRELESS POWER GENERATION AND TRANSMISSION TECHNIQUE}

Family unit gadgets create generally little magnetic fields. Therefore, chargers hold gadgets at the separation important to initiate a present, which can just happen if the curls are near one another. Since an attractive field spreads every way, making a bigger one would squander a considerable measure of vitality [9]. An effective approach to exchange control between loops isolated by a couple of meters is that we could expand the separation between the curls by adding resonance to the condition. A decent approach to comprehend resonance is to consider it as far as sound. A question's physical structure - like the size and state of a trumpet - decides the recurrence at which it normally vibrates. This is its resonant recurrence. It's anything but difficult to motivate articles to vibrate at their full recurrence and hard to inspire them to vibrate at different frequencies. This is the reason playing a trumpet can make an adjacent trumpet start to vibrate [10]. The two trumpets have the same resounding recurrence. Enlistment can occur little distinctively if the electromagnetic fields around the loops resound at a similar recurrence. The hypothesis utilizes a bended curl of wire as an inductor. A capacitance plate, which can hold a charge, joins to each finish of the loop as appeared in Figure 1. As power goes through this curl, the loop starts to reverberate. Its thunderous recurrence is a result of the inductance of the curl and the capacitance of the plates [11].

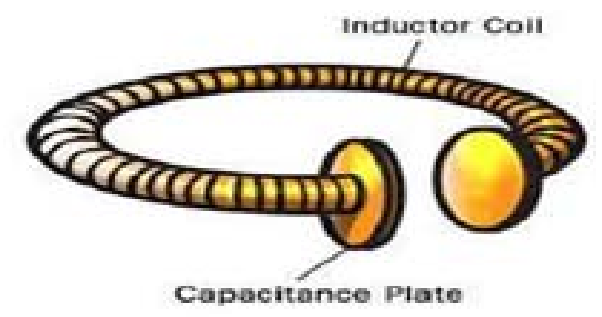

FIGURE I. CAPACITANCE PLATES WITH CURL

Power, going along an electromagnetic wave, can burrow from one loop to alternate as long as they both have the same resonant recurrence [12]. In a short hypothetical investigation they exhibit that by sending electromagnetic waves around in an exceedingly precise waveguide, transitory waves are created which convey no vitality. A transient wave is close field standing wave showing exponential rot with separate. On the off chance that a legitimate full waveguide is brought close to the transmitter, the evanescent waves can enable the vitality to burrow (particularly transient wave coupling, what might as well be called burrowing to the power drawing waveguide, where they can be corrected into DC control. Since the electromagnetic waves would burrow, they would not proliferate through the air to be ingested or scattered, and would not disturb electronic gadgets. For whatever length of time that the two loops are out of scope of each other, nothing will happen, since the fields around the curls aren't sufficiently solid to influence much around them. So also, if the two curls reverberate at various frequencies, nothing will happen [13]. Be that as it may, if two resounding loops with a similar recurrence get inside a couple of meters of each other, surges of vitality move from the transmitting curl to the accepting loop. As per the hypothesis, one loop can even send power to a few getting curls, as long as they all resound at an indistinguishable recurrence from appeared in Figure 2. The specialists have named this non-radiative vitality exchange since it includes stationary fields around the curls as opposed to fields that spread every way [14]. 


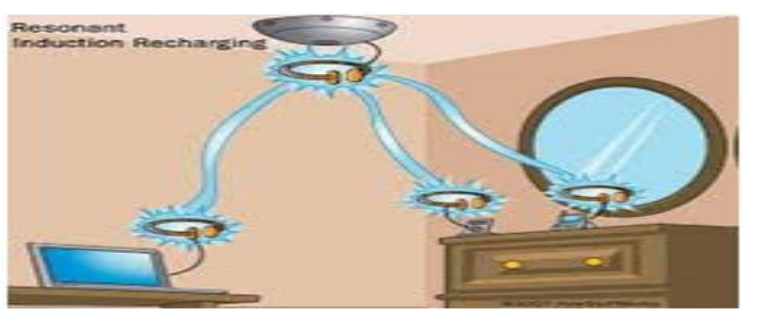

FIGURE II. POWER TRANSMISSION TO MULTIPLE CURLS

As indicated by the hypothesis, one loop can energize any gadget that is in go, as long as the curls have the same full recurrence. "Full inductive coupling" has enter suggestions in taking care of the two principle issues related with non resonant inductive coupling and electromagnetic radiation, one of which is caused by the other; separation and productivity [15]. Electromagnetic enlistment chips away at the rule of an essential curl producing a transcendently attractive field and an optional loop being inside that field so a current is actuated inside its loops. This causes the moderately short range because of the measure of energy required to create an electromagnetic field. Over more noteworthy separations the non-resonant enlistment strategy is wasteful and squanders a great part of the transmitted vitality just to expand [16]. This is the place the resonance comes in and helps productivity significantly by "burrowing" the magnetic field to a collector loop that resounds at a similar recurrence. Not at all like the different layer optional of a non-resonant transformer, such accepting loops are single layer solenoids with firmly divided capacitor plates on each end, which in mix enable the curl to be tuned to the transmitter recurrence in this manner dispensing with the wide vitality squandering "wave issue" and permitting the vitality used to concentrate in on a particular recurrence expanding the range.

\section{PROBLEM DESCRIPTION}

We accept $\mathrm{N}$ sensors arbitrarily appropriated with an equivalent likelihood in a detecting region. Nc groups are predecided for the system. Along these lines, $\mathrm{Nc} \mathrm{CHs}$ are haphazardly looked over all the sensors in light of a likelihood $\mathrm{Nc} / \mathrm{N}$. This can share the weight of workload of being CHs for each sensor. At that point, the rest as non- $\mathrm{CH}$ sensors pick one $\mathrm{CH}$ which is nearest to shape bunches. By and large, each bunch has $(\mathrm{N} / \mathrm{Nc})$ sensors. What's more, each $\mathrm{CH}$ has $(\mathrm{N} / \mathrm{Nc}-1)$ non-CH sensors. As appeared in

Figure 3, non-CH sensors send specifically information to their own CHs. Each $\mathrm{CH}$ forms the gathered information inside its group also, sends a specific number of tests to the BS for information recreation following the Wavelet-based information pressure calculation.

As said the past areas, the system is isolated into Nc group. Figure 1 demonstrates the bunched arrange in general with the $\mathrm{BS}$ outside the detecting zone. For the most part, all non-CH sensors send their information to the CHs. All the CHs forward all the got information including their own to the BS.

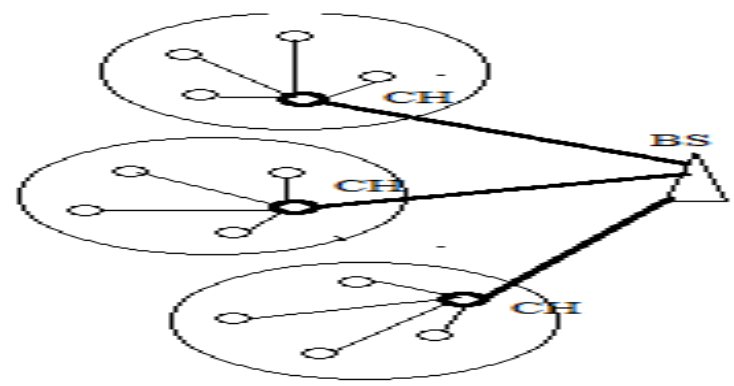

FIGURE III. CLUSTER HEAD WITH BASE STATION

In the proposed calculation, each $\mathrm{CH}$ makes one Wavelet sub-framework $\varphi \mathrm{j}$ with measurement $(\mathrm{mj} \times \mathrm{mj})$, where $\mathrm{mj}$ is the number of sensors in the jth bunch. Information gathered at each bunch is increased to a sub-lattice in which, every one of the passages are made by the Matlab charge haarmtx(mj). A huge extent of the flag vitality is centered on the principal huge coefficients, given as $\mathrm{K}$ coefficients. Just these coefficients are sent to the BS from all the CHs. The rest of the changed coefficients can be considered as zeros. At the transmission time only $\quad K=\Sigma_{j}$ large coefficient will sent so that it save lot of power in transmission sense.

Transformed coefficient is shows in the form of $\mathrm{zj}$, and it is represented as $z j=\phi j x j$. In this method number of sensors is propositional to the number of Major coefficient $(\mathrm{kj} / \mathrm{K})=$ $(\mathrm{mj} / \mathrm{N})$. After transmitting the data to base station it can calculate the received data into original form like $x^{\prime} j=\phi T j z j$

The proposed calculation can likewise function admirably with adaptation to non-critical failure in the system since all sensors alternate to wind up CHs. Breakdown hubs are not picked after each round of picking $\mathrm{CHs}$ or non- $\mathrm{CH}$ sensor hubs. The flaws of the system or on the other hand sensor hubs could be recognized and recuperated by blame tolerant calculations for grouped systems or for tree based systems. Broke down hubs are detached yet could be utilized for transferring information in the system if conceivable. This could be an open work for our future research.

\section{A. Optimizrd Information Compression Algorithm}

1. In this algorithm the network is divided into various clusters name as $\mathrm{Nc}$

2. Every cluster head work is to collect data from the all other node of the cluster including its own data.

3. After collecting all the data it multiplies it into certain function.

4. Certain Sample of that function called as major coefficient is sent to the base station as transmitting data.

5. The transmission of the data to base station can be perform into two ways like either the data is directly sent to the base station or it is sent with the help of intermediate nodes.

6. At the receiver point the receiver collect the data from Nc clusters and then wrap out the data with the help of reverse method and get the original data. 
7. With the help of the method less data is transmitted through the channel and it save the lot of power of the nodes and cluster head and ultimately the power of network.

\section{B. Power Consumption Analysis}

Network uses the power for transmitting and receiving the data and these are denoted by PT1 and PT2 respectively.

$$
\begin{gathered}
\mathrm{P}_{\mathrm{T} 1}=\mathrm{P}_{\mathrm{T}}+\mathrm{Pa}(\mathrm{d}) \\
\mathrm{P}_{\mathrm{T} 2}=\mathrm{P}_{\mathrm{R}}
\end{gathered}
$$

$\mathrm{P}_{T}$ and $\mathrm{P}_{R}$ are the power used for the part of non transmission and non receiving processes. $\mathrm{Pa}$ is the actual power consumed and it is the function of distance. This manuscript calculate the two type of power consumption first used in transmission between sensors and cluster head and second which is used in transmission from cluster head to base station.

\section{A) Psc power consumption calculation}

According to the network distribution in clusters $\mathrm{Nc}$. The network consist of one cluster head and $(\mathrm{N} / \mathrm{Nc}-1)$ sensors nodes.

$$
\mathrm{Psc}=\mathrm{Nc}(\mathrm{N} / \mathrm{Nc}-1) \mathrm{E}[\mathrm{r} \alpha]
$$

In the above equation $r$ is behave like a random value of distance between the Cluster head to sensor nodes and alpha denotes the pathway loss exponent.

According to the paper the value of the $\mathrm{E}[\mathrm{r} \alpha]$ can be calculated as

$$
\mathrm{L}^{2} / 2 \pi \mathrm{Nc}
$$

so that the power consumption of Psc is as

$$
\operatorname{Psc}=(\mathrm{N} / \mathrm{Nc}-1) \mathrm{L}^{2} / 2 \pi
$$

B) Pcb power consumption calculation

Major coefficient of the network is calculated as

$$
\mathrm{K}=\mathrm{Nc} \mathrm{kj}
$$

The Power consumed by the transmission for cluster head to base station is as

$$
\mathrm{Pcb}=\mathrm{K} \mathrm{E}\left[\mathrm{d}^{2}\right]
$$

According to paper the total power is calculated as

$$
\text { Ptotal }=(\mathrm{N} / \mathrm{Nc}-1) \mathrm{L}^{2} / 2 \pi+\mathrm{KL}^{2} / 6
$$

\section{SiMULATIONS AND RESUltS}

We think about both regular sorts of systems, square detecting territory with measurement $150 \times 150$. In this system, there are 1500 sensors arbitrarily sent. K-implies and LEACH are likewise conveyed to gather information and to contrast and our examination comes about. Genuine sensor readings gathered from Sensor scope are utilized for recreating. We assess the aggregate vitality utilization for the systems and give the execution of the proposed calculation with the information.

Figure 4 represents the line graph between power consumption in Intra cluster Communication and number of clusters in the network. As graph shown the three different curve for LEACH, K-means and Proposed Optimized Information Compression algorithm. Power consumption in LEACH is higher than the K-Means and OICA algorithms. When we look for the K-Means and OICA curves it is look similar that means proposed algorithm give slightly better results than K-means algorithm.

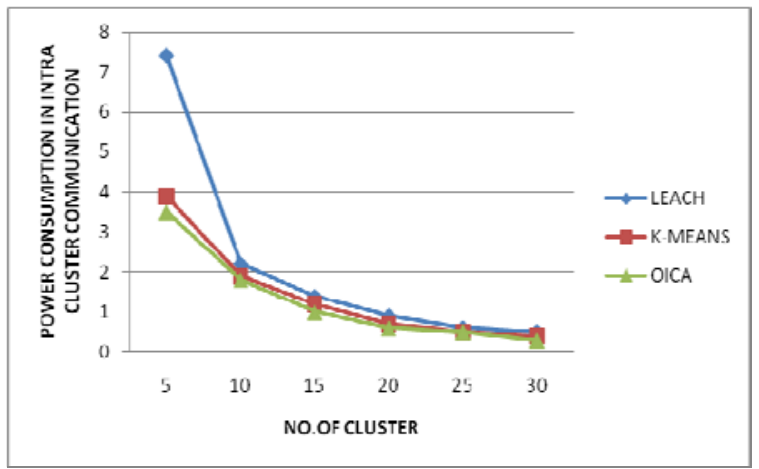

FIGURE IV. POWER CONSUMPTION IN INTRA CLUSTER COMMUNICATION VS NO. OF CLUSTERS

Figure 5 represents the line graph between power consumption in communication to base station and number of clusters. This line graph shows the variation of power consumption between the LEACH, k-means and proposed algorithm as the number of clusters increases. According to the graph proposed algorithm behave better than other to algorithm in respect of power consumption in communication to base station.

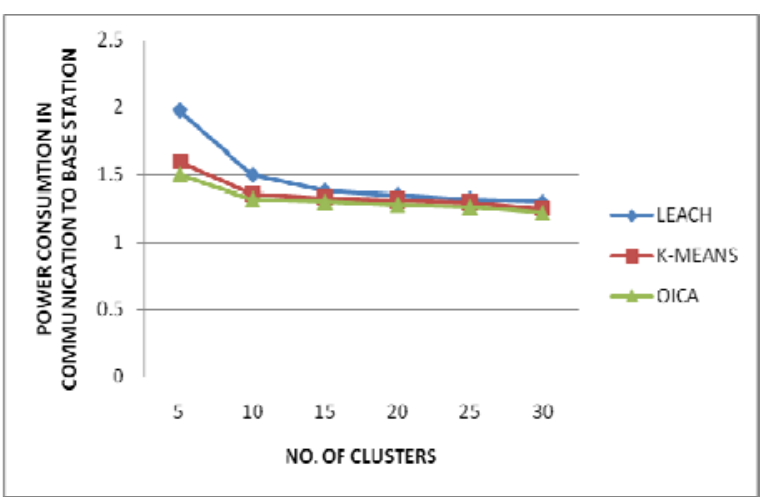

FIGURE V. POWER CONSUMPTION IN COMMUNICATION TO BASE STATION VS NO. OF CLUSTERS 
Figure 6 shows the graph in which different temperature reading are displayed. Each and every sensor gives the recorded reading to the cluster head of the each cluster. This step is the basic or traditional step which is followed by every sensor node in the network.

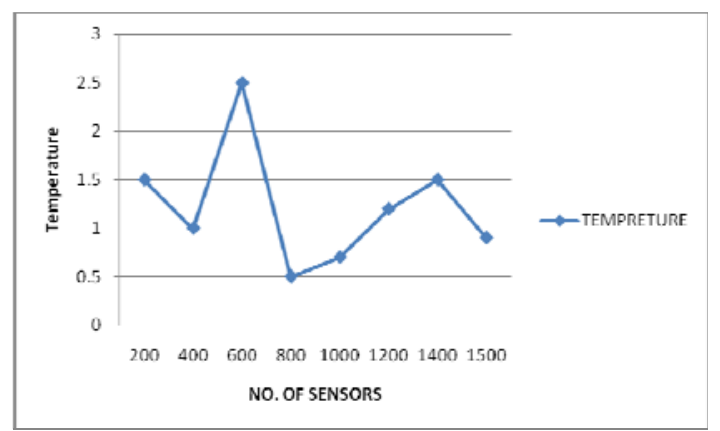

FIGURE VI. TEMPERATURE VS NO. OF SENSORS

Figure 7 represent the line graph of optimized transformed coefficient which is transfer to the base station instead of all the readings will be transfer to base station. That is the job of cluster head to create the coefficient and then transformed to base station so that lot of power would be saved.

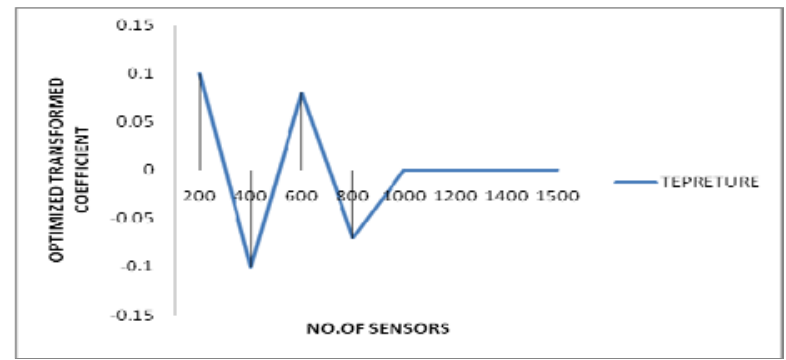

FIGURE VII. OPTIMIZED TRANSFORMED COEFFICIENT VS NO. OF SENSORS

\section{CONCLUSION}

This manuscript proposed optimized information compression algorithm (OICA) for bunched WSNs to decrease vitality utilization in gathering tangible information. In light of the way that all information vitality centered in moderately little quantities of vast coefficients in the changed vector, we just send the vast coefficients to the BS for the flag recuperation process. These coefficients are mapped at the BS to recoup all tactile readings from the system. We examined and planned either the intra-bunch vitality utilization or the aggregate devoured vitality for the system to transmit information. Reenactment comes about are accommodated both expended estimation and the optimized information compression technique. We recommend an ideal case for the systems to utilize multi-bounce if many groups are connected. Second proposed method to resolve the power problem of wireless sensor network is also give significant results with the help of this concept sensor are free with power problem. In future work, we will ponder the limit for the quantity of bunches in both commotion and silent situations.

\section{REFERENCES}

[1] T. Arampatzis, J. Lygeros, and S. Manesis, "A survey of applications of wireless sensors and wireless sensor networks," in Intelligent Control, Proceedings of the 2005 IEEE International Symposium on, Mediterrean Conference on Control and Automation, pp. 719-724, IEEE, 2005.

[2] M. J. Kim, "CHEF: Cluster Head Election mechansim using Fuzzy logic in Wireless Sensor Networks", Proc. of ICACT, pp. 654- 659, 2008.

[3] M. Bhardwaj and A. Ahlawat, "Reduce Energy Consumption in Ad hoc Network with Wireless Power Transfer Concept ", International Journal of Control Theory and Applications, vol. 10, issue 13, 2017.

[4] M. Bhardwaj, "Enhance life Time of Mobile Ad hoc Network using WiTriCity and Backpressure Technique", 1877-0509 (C) 2015 The Authors. Published by Elsevier B.V. This is an open access article under the CC BY-NC-ND license, doi: 10.1016/j.procs.2015.07.447.

[5] M. Bhardwaj, "Selection of Efficient Relay for Energy-Efficient Cooperative Ad Hoc Networks" American Journal of Networks and Communications. Special Issue: Ad Hoc Networks, vol. 4, no. 3-1, pp. 511, 2015.

[6] S. Nikoletseas, "An experimental evaluation of wireless power transfer protocols in mobile ad hoc networks", IEEE Wireless Power Transfer Conference (WPTC), pp.1- 3, 2015.

[7] M. B. Makaa,, "Analysis of WPT system using rearranged indirect-fed method for mobile applications", IEEE Wireless Power Transfer Conference (WPTC,) pp.1- 4, 2015.

[8] Z. Han, "Wireless Charging Technologies: Fundamentals Standards and Network Applications", Communications Surveys \& Tutorials IEEE, vol. 18, pp. 1413-1452, ISSN 1553-877X.

[9] M. Bhardwaj and A. Bansal,"Energy Conservation in Mobile Ad Hoc Network Using Energy Efficient Scheme and Magnetic Resonance", Advances in Networks. Special Issue: Secure Networks and Communications, vol. 3, no. 3-1, pp. 34-39, 2015.

[10] J. Kim, J. Jang, "AODV based EnergyEfficient Routing Protocol for Maximum Lifetime in MANET", IEEE Conference on Telecommunications, pp. 1-212, 2006

[11] L. Guo, Y. Liu, T. Jiang, "Energy Efficient on-demand MultipathRouting Protocol for Multi-hop AdHoc Networks", IEEE Symposium on Spread Spectrum Techniques and Applications, pp. 1-820, 2009.

[12] G. Ferrari, S.A. Malvassori, "Physical Layer-ConstrainedRouting in Adhoc Wireless Networks: A Modified AODV Protocol with Power Control" IEEE International Workshop on Wireless Ad hoc Network, pp23-27, 2005.

[13] R. C. Shah, J. M. Rabaey, "Energy awarerouting for low energy ad hoc sensor networks", IEEE Wireless Communications and Networking, pp. $1-931,2002$.

[14] M. Bhardwaj, A. Singh, "Power Management of Ad Hoc Routing Protocols Using Mobility Impact and Magnetic Resonance", Advances in Networks. Special Issue: Secure Networks and Communications, vol. 3, no. 3-1, pp. 27-33, 2015.

[15] S. Nikoletseas, P. T. Raptis, "Interactive Wireless Charging for Energy Balance", IEEE Distributed Computing Systems (ICDCS), pp. 262-270, ISSN 1063-6927, 2016.

[16] M. Bhardwaj, A. Pathak, "Optimum Experimental Results of AODV, DSDV \& DSR Routing Protocol in Grid Environment", Procedia computer science, 3rd International Conference on Recent Trends in Computing, vol 57, pp. 1342-1350, 2015. 